\title{
Zwei Fälle von malignem Fibromyom des Uterus ${ }^{1}$ ).
}

Von

\author{
Dr. Kynoeh, \\ Prof. der Geburtşhülfe u. Gynäkologie an der St. Andrews Universitäit, Schottland.
}

(Mit 9 Figuren im T'ext.)

Die Verbindung von Uterus-Fibromyom mit bösartiger Veränderung im glejchen Organ ist ein Gegenstand von grossem path. Interesse und klinischer Wichtigkeit. Solch eine Combination wurde früher als sehr selten erachtet, da man annahm, dass das Vorhandensein eines Fibroids die Entwicklung eines Carcinoms ausschliesse. Jedoch das Studium der neueren Statistiken über degenerative Veränderungen der Fibromyome zeigt, dass diese Tumoren, insofern sie adenomatöse Hypertrophien des Endometriums bewirken, die Entwicklung von Adenocarcinomen begünstigen. Weitaus die grösste Anzahl sogen. carcinomatös degenerirter Fälle von Fibromyomen sind Myome bei welchen das Carcinom im Endometrium entstanden ist. Eine kleinere Anzahl von Fällen jedoch, wie von Recklinghausen beschrieben, sind solche, welche in der Entwicklung von Adenomyomen entstanden sind. Dass ein fibröser Tumor primär carcinomatös degeneriren kann, wird noch von vielen Autoritäten bezweifelt. Die meisten, bisher zu diesem Zweck veröffentlichen Fälle haben nicht die nöthige Beweiskraft gehabt, um die Möglichkeit einer Carcinomentwicklung von tiefer gelegenen Drüsengängen her, ausschliessen zu können. Der von Liepmann beschriebene Fall ist vielleicht der einzige, welcher jeder Kritik Stand hält.

Er beschreibt einen Fall von Carcino-Myom mit Lungen- und Drüsenmetastasen, welcher vielleicht die Möglichkeit einer direkten Umwandlung von Myomgewebe in Carcinom glaubhaft erscheinen lässt. Zukïnftige Untersuchungen werden wahrscheinlich zeigen,

1) Aus dem Englischen übersetzt von Dr. Otto Seitz, Frauenarzt, München. 
dass diese Combination viel häufiger ist, als allgemein angenommen, und dass wahrscheinlich ein direkter causaler Zusammenhang zwischen beiden besteht. Piquand stellt fest, dass Carcinom des Corpus uteri 8 bis 9 mal öfter vorkommt bei Frauen mit Fibromen, und Winter hält diese Combination auch für richtig, denn er macht auf die Thatsache aufmerksam, dass Uteruscarcinom in Verbindung mit Fibrom in einem solchen Verhältniss vorkommt, dass 23 Carcinomfälle des Corpus uteri auf 13 Fälle der Cervix treffen. Blandsutten jedoch ist noch nicht überzeugt von dem causalen $\mathrm{Zu}$ sammenhang und glaubt, dass erst spätere klinische und pathologische Beobachtungen den Beweis erbringen können. Von 63 von ihm operirten Fibromyom-Fällen waren 8 complicirt mit Carcinom der Uterussehleimhaut. Noble hat eine Sammelstatistik von insgesammt 2374 Fällen zusammengestellt um die Complicationen und Degenerationen der Fibromyome zu zeigen. Davon waren $43=1,8$ pCt. Corpus-Carcinome, im Gegensatz zu $16=7$ pCt. Cervix-Carcinomen. Da das gewöhnliche Verhältnis von CervixCarcinom zu Corpus-Carcinom ungefähr wie $10 \mathrm{zu} 1$ ist, so hätten wir also $430 \mathrm{zu}$ erwarten gehabt, statt nur 16 . Es muss jedoch bemerkt werden, dass die augenscheinliche Seltenheit von CervixCarcinom in Verbindung mit Fibromyom, sehr oft durch die, bei Fibromyomen vorkommende Sterilität bedingt ist. In Folge von Sterilität gelten ja auch die Cervixeinrisse als prädisponirender Factor. Noble hat ferner 4880 Fälle von Uterus-Fibromen gesammelt; davon waren 63 Fälle Cervix-Carcinome, 75 CorpusCarcinome, im Ganzen 138 Carcinom-Fälle von diesen 4880 oder 2,8 pCt. Wenn wir diese letzteren Fälle eintheilen, so finden wir Cervix-Carcinome 9 pCt. und Corpus-Carcinome 1,54 pCt. Noble glaubt daraus schliessen zu müssen, dass Fibrom-Tumoren eine Prädisposition zum Corpus-Carcinom abgeben.

In Folgendem soll ein Fall beschrieben werden von Adenocarcinom der Uterussehleimhaut, in Verbindung mit interstitiellen Fibromyomen des Uterus:

Frau G. 49 J. alt, Haushälterin, liess sich am 1. October 1902 an der Gynäk. Abth. des Kgl. Dundee-Krankenh. aufnehmen. Sie klagte über einen bereits 9 Monate dauernden heftigen Ausfluss. Derselbe begann als sebr beftige Menstruation und währte die letzten 14 Tage ununterbrochen. Menstruirt ward die Pat. zum 1. Mal mit 13 Jahren, alle 28 Tage regelmässig aber immer sehr reichlich. Vollständig ausgeblieben war ihr die Periode im April, Mai und Juni 1901, kam aber dann stärker als je zuvor. Sie hatte keine ausgetragenen Kinder, sondern 3 im 3. Nonat erfolgte Fehlgeburten vor 20 Jahren. Sie hatte weder 
Schmerzen noch Blasenbeschwerden, schlief aber schlecht und laborirte an Kopfweh, manchmal mit Uebelkeit und Erbrechen. P. war anämisch und man hörte ein systolisches Geräusch über der Herzgrube, mit verschiedenen starken Herztönen. Der Uterus war durch ein interstitielles Fibromyom gleichmässig vergrössert; dasselbe reichte bis in die Mitte zwischen Nabel und Symphyse, und fühlte sich durch das hintere Scheidengewölbe höckerig an. Die Dämpfung betrug $4 \% \frac{1}{2}$ Zoll im Durchmesser. In Anbetracht der Anämie der Pat. bekam sie Ergotin und Blaud'sche Pillen $3 \times$ tägl. Am 18. October Chloroformnarkose; Erweiterung und Curetage der Uterushöhle zeigte ein stark verdicktes Endometrium. Der Uterus wurde mit Gaze tamponirt, welche am 20. wieder entfernt w urde.

Histologische Untersuchung der Präparate: Man sah meistens gut ausgebildete Drüsen, derèn Hoblräume mit papillären Wucherungen ausgefüllt waren. An anderen Stellen sab man unregelmässig verzweigte schmale Bindegewebszüge, zum Theil mit epithelialem Ueberzug. Das Epithel war an manchen Stellen einschichtig von typischer Form, aber sehr oft fanden sich auch mehrschichtige theils rundliche, theils squamöse Epithelien. Die Kerne waren meistens oval, unregelmässig in Grösse und Lagerung, viele gequollen, meistens granulirt. Andere bildeten grosse Blasen, dunkelgekörnte Zellen enthaltend. Die Kerne zeigten vielfach frische Theilungsfiguren mit der Theilungsachse parallel zur Längsachse. Nur ca. 2 Drüsenlumina erhielten massenhaft atypische, Schuppenepithelien ähnliche, meistens dunkelgekörnte Zellwucherungen. Die atypische Anordnung der Drüsenepithelien zusammen mit der starken Proliferation und mit der Neigung zu papillären Wucherungen berechtigte zu der Diagnose: beginnendes Adenocarcinom. Die Pat. wurde von der Nothwendigkeit einer weiteren Operation verständigt, verweigerte aber die Erlaubniss. Erst am 5. Mai 1905 kam sie wieder, da die Blutmengen ununterbrochen fortgedauert hatten. Am 16. Mai Bauchschnitt in Chloroformnarkose. Das linke Ovarium erwies sich als multiloculäre Cyste; beide Ovarien wurden entfernt.

Am 28. Juni wurde die Pat. in gutem Zastand entlassen; ungefähr 6 Monate nach der Operation zeigten sich abermals Unterleibsbeschwerden, und im April 1906 starb die Pat. unter den Symptomen des Bauchkrebses.

Pathologisch anatomische Beschreibung des Uterus: (Fig. 1). Der Uterus bildete einen unregelmässigen ungefähr $9 \mathrm{~cm}$ dicken und $17 \mathrm{~cm}$ langen Tumor. Die Oberfläche war höekerig in Folge von Myomen, 3 davon waren gestielt, $2 \mathrm{im}$ Fundus und einen verschloss die Cervix. Beim Aufschneiden des Uterus fand sich die normale Uterusmuskulatur im Fundus $1 / 2 \mathrm{~cm}$ dick. Unterhalb befanden sich 2 Fibromyome von harter Consistenz $(31 / 2$ mal $2 \mathrm{~cm}$ und $3 \mathrm{mal} 3 \mathrm{~cm})$. Andere Myome durchsetzten die vordere Uteruswand. Das Endometrium war der Sitz einer sehr starken fingerdicken carcinomatösen Wucherung, welche ihre Ausläufer allseitig in die Muskulatur ausschickte. Das ganze Endometrium schien gleichmässig ergriffen und zwar rechts hinunter bis zur Cervix. In 
den oberen Partien der Utẽrushöhle reichten die Wucherungen bis $1 \mathrm{~cm}$ unter den Peritonealüberzug. Zwisehen dem Krebs und der Muskelwand war ein sehr deutlicher Farbenunterschied.

Histologische Untersuchung: (Fig. 2 und 3). Es fand sich keine Spur von normaler Schleimhaut, da die ganze Uterushöhle vollständig von der Neubildung ausgefüllt war. Durchschnitte durch den Rand der Neubildung zeigten ein typisches Adeno-

Figur 1.

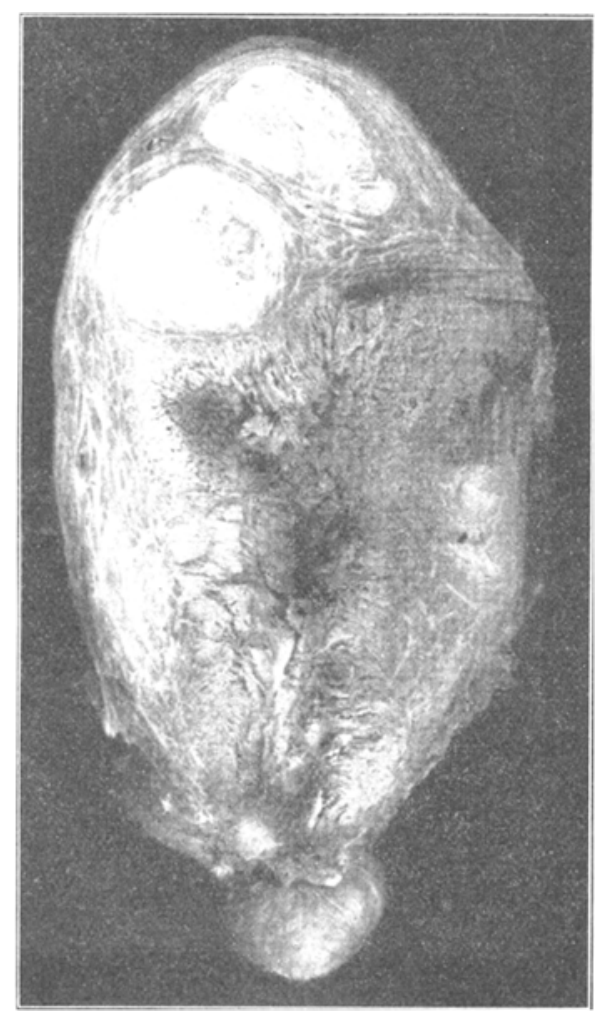

carcinom, bei welchem der Drüsencharakter besonders gut gewahrt schien. Auswüchse aus mehrschichtigen Epithelzellen bestehend, durchdrangen die Uteruswand nach allen Richtungen. Die Epithelien waren vielfach regelmässig geformt, mit deutlichen dunklen Sternen. Aber an den meisten Stellen war es ein vielschichtiges squamöses, solide Lappen bildendes, Epithel, das von sehr spärlichem Binde- 
258 Kynoch, Zwei Fälle ron malignem Fibromyom des Uterus.

Figur 2.

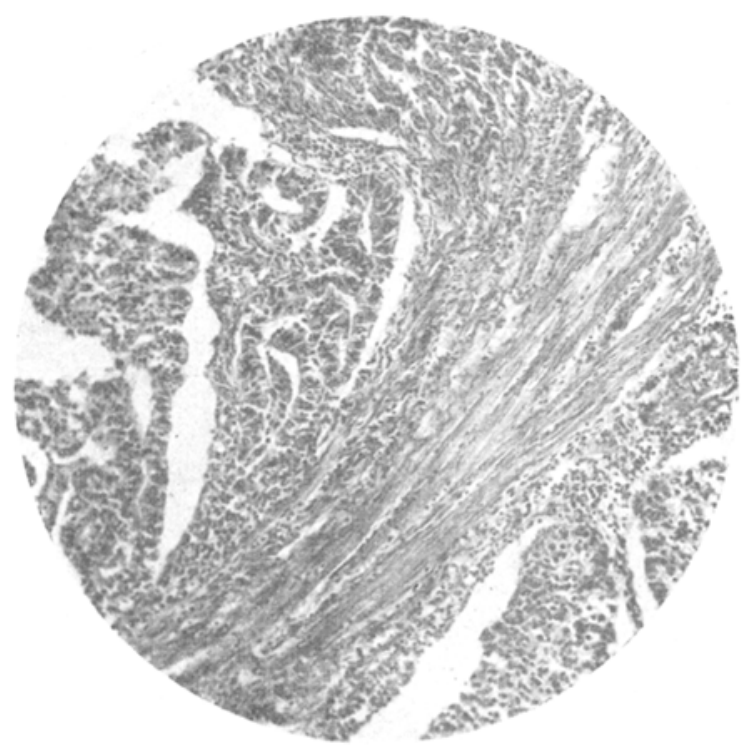

Figur 3 .

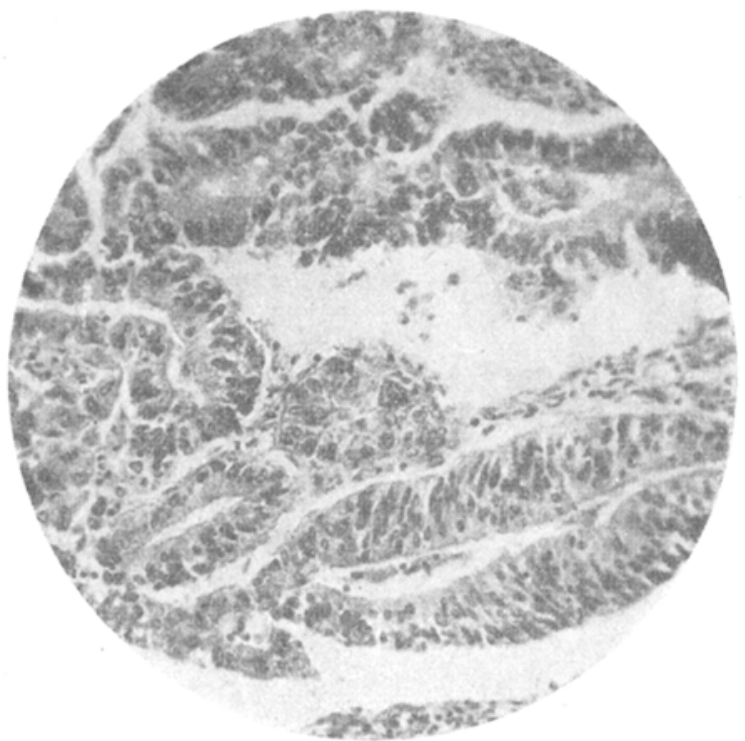

gewebe umgeben war. Das Stroma und das umgebendo Muskclgewebe zeigte Rundzelleninfiltration.

Fibrom complicirt mit Sarkom.

Während, wie schon erwähnt, die Umwandlung von Fibromyom 
in Carcinom noch stellenweils als unsicher eräahtet wird, sind jetzt eine genügend grosse Anzahl von Fällen berichtet worden, die die Möglichkeit einer sarkomatösen Umwandlung zeigen. Nichtsdestoweniger macht auch hier das Studium dieser Beziehungen Schwierigkeiten, und erfordert Vorsicht.

Gusserow in "Die Neubildungen des Uterus" sagt über die sarkomatöse Degeneration der Fibromyome folgendes:

„Bis jetzt ist noch kein Fall von Uterus-Sarkom beschrieben, der eine Kapsel besass wie die meisten Fibromyome. Das ist wichtig in Bezug auf die Frage der Verwandlung von. Fibromyom in Carcinom". Aber wir wissen jetzt, dass mehrere solche Fälle beschrieben worden sind. Fälle von diffusem nicht eingekapselten Uterussarkom sind meistens primär, kommen bei jugendlichen Pat. vor und die Tumoren bestehen noch nicht lange. Ferner müssen wir unterscheiden zwischen sarkomatöser Veränderung und zwischen Entzündung eines Fibroms. Das histologische Bild kann so ähnlich sein, dass der Patholog nur schwer unterscheiden kann, ob der Fall malign ist oder nicht.

Ein gewisser Routh z. B. demonstrirte vor der Londoner Gynäkologischen Gesellschait ein Präparat, welches er als MyxoSarkom des Uterus beschrieb. Aber schon das pathol. anat. Comitée dieser Gesellschaft erklärte das Präparat als ein durch hochgradige Entzündung erweichtes Fibromyom. Die Veränderungen, welche in einem entzündeten Fibromyom in Bezug auf Muskelfasern und Bindegewebe vor sich gehen, können leicht als Beweis der Malignität ausgelegt werden. Es kann deshalb die Wichtigkeit des Sectionsberichtes nach Fibromyom-Operationen mit Verdacht auf Sarkom nicht hoch genug geschätzt werden. Bezüglich der Häufigkeit dieser Verbindung glaubt Winter, dass wenn man alle Myome systematisch untersuchen würde, man 4 pCt. Sarkome darunter finden würde. Hier folgt eine Procentberechnung:

$$
\begin{aligned}
& \text { v. Franqué }=2,3 \text { pCt. } \\
& \text { Fehling }=4 " \\
& \text { Hauber }=3 " \\
& \text { Piquand }=2 "
\end{aligned}
$$

Sarkomatöse Veränderungen der Fibrome kann es unter folgenden Umständen geben:

1. Im selben Uterus können zwei verschiedene Tumoren sein, insofern der eine ein Sarkom und der andere ein Fibrom ist. 
2. Das Sarkom kann im Endometrium oder einem anderen Theil des Uterus entstehen und in Folge dessen in ein Fibrom hineinwachsen.

3. Ein Sarkom kann in einem schon vorhandenen Fibromyom neu entstehen.

4. Die Zellen, aus welchen ein Fibromyom besteht, können event. einen malignen Charakter annehmen.

Dann kann es sehr schwer sein, Beispiele der letaten beiden Art von einander zu unterscheiden. Der folgende Fall von Sarkom in Fibromyom würde wahrscheinlich unter die letztere Rubrik kommen, obwohl unmöglich sicher festzustellen ist, ob die Sarkomzellen im Muskel- oder im Bindegewebe entstanden sind. Der Fall zeigt alle Merkmale der Malignität, nämlich Metastasen und tödtlicher Ausgang ein Jahr nach der Operation.

Frau Y., 48 Jahr alt, kam am 8. 2. 05 auf die Gynäk. Abth. des kgl. Dundee. Krankenhauses. Sie klagte über zu starke Menstruation seit 11 Jabren und seit der gleichen Zeit hatte sie einen Tumor im Leibe bemerkt. 2 Monate vor ihrer Aufnahme im Spital hatte sie sehr starke Blutungen, welche fast ununterbrochen fortdauerten; dadureh wurde sie äusserst blutarm. Ausser etwas Kopfweh vor der Menstruation hatte sie nie viel Schmerzen gehabt, früher war sie immer gesund. Der Vater ist am Leben und gesund, die Mutter starb, 67 Jahre alt, an Herzleiden. 4 Schwestern und 2 Brüder sind gesund. Die Yenstruation hatte mit 14 Jahren begonnen, stellte sich regelmässig und in normaler Quantität alle 28 Tage ein, bis vor 11 Jahren. Seit dieser Zeit hatte sie Menorrhagien mit Dysmenorrhoe wie oben bemerkt. Sie hatte 3 ausgetragene Kinder, von denen das älteste 28 und das jüngste $25 \mathrm{Jahre} \mathrm{zăhlte.}$

Bei der Aufnahme war die Pat. anämisch und schwächlich; Herz und Lungen waren normal. Das spec. Gewicht des Urins betrug 1020; Eiweiss war nicht nachweisbar; Puls 90 und sehr schwach. Bei der Untersuchung des Bauches fand sich ein runder harter vom Becken bis $1 \frac{1}{4}$ Zoll über den Nabel reichender Tumor. Die Cervix hing mit dem Tumor zusammen und war für einen Finger durchgängig.

Der Tumor wurde als ein interstitielles Fibrom des Uterus diagnosticirt. Wegen der grossen Schwäche der Pat. gab man ihr $3 \mathrm{Mal}$ täglich Haematogen und Portwein. Am 1. 3. wurde der Uterus unter Chlorof.Narkose durch supravaginale Amputation exstirpirt. Beide Ovarien wurden, da gesund, zurückgelassen. Pat. machte eine gute Reconvalescenz durch und wurde am 29.4. gesund entlassen. Dieses Wohlbefinden dauerte 6 Monate, da fand sich plötzlich eine orangengrosse harte Geschwulst an der Vorderseite des rechten Ellbogens (Fig. 9). Sie wurde im Januar 1906 entfernt. Der Gesundheitszustand besserte sich aber nicht, überall über dem Abdomen fanden sich Knoten und nach verschiedenen Anfällen von Blutspeien starb die Pat. im Juni 1906 unter den Symptomen einer recurrirenden malignen Erkrankung. Die Section wurde nicht gestattet. Der Fibromyom-Uterus wurde nun sorgfältig untersucht. 
Präparat No. 4280 .

Der Uterus ist ein grosser, harter, höckeriger Tumor, die Cervix wurde im Niveau des Tumors abgetragen, und die Uterusöffnung erscheint durch das Tumorgewebe erweitert. Der seröse Ueberzug ist zart und frei von Adhäsion. Der gehärtete Tumor misst 5 Zoll Höhe, 9 Zoll Dicke, 7 Zoll Breite. Seitlich aufgeschnitten findet sich ein solider runder Tumor mit einer dünnen Muskelkapsel, deren unterer Abschnitt erweicht ist. Auf der Schnittfläche befindet sich keine Spur von Uterushöhle, dieselbe erscheint jedoch an der vorderen Seite des Tumors.

Die Schleimhaut der vorderen Uteruswand, ist soweit sichtbar, zart und durch den Druck des Tumors auf einen halben Zoll reducirt. Der ganze Eindruck war der eines vereiterten grossen Fibromyoms und gab zu keinerlei Verdacht Anlass: desshalb wurde die mikroskopische Untersuchung erst ein Jahr nach dem Auftreten der sesundären Erscheinungen in Bauch und am Arm der Patientin gemacht. Bei der späteren Untersuchung zeigte das. Centrum des Tumors ein weisses, hartes, glänzendes Aussehen, so wie man es bei typischen Fibromyomen des Uterus findet. Im Gegensatz dazu war die Uteruswand, soweit sie vom Tumor durchsetzt war, ganz anders, nämlich blutig verfärbt, gelblich und bei Berührung sehr weich. In der Mitte dieses weicheren durchbluteten Gewebes hat jedenfalls ein Durchbruch stattgefunden. Offenbar ist also der Tumor durch seine Kapsel in der Riehtung der Uterushöhle durchgebrochen, dieselbe mit bröcklichen unregelmässigen Massen ausfüllend. Man kann also 3 Theile an diesem Tumor unterscheiden:

a) den harten fibrösen Theil,

b) den Fibromyom-Theil,

c) den durchbluteten und sehr weichen Theil.

Der secundäre Tumor am Arm der Pat. war unregelmässig gelappt und fast $3 \frac{1}{2}$ Zoll dick und 3 Zoll breit. Der Durchschnitt theilweise hart und theilweise weich, weisslich und stellenweise gestreift. An einer Stelle sah man Spuren von derberem Bindegewebe.

Histologische Untersuchung des grossen Tumors.

Die Schleimhaut:

Sie ist zu einer ganz dünnen Gewebslage reducirt, das Oberflächen-Epithel ist meistens zerstört; wo es erhalten ist, finden sich niedrige kubische Zellen. Die Drüsen nach Zahl und Grösse vermindert, das Epithel beschädigt, das Stroma zeigt etwas klein- 
262 Kynoch, Zwei Fälle von malignem Fibromyom des Uterus.

\section{Figar 4.}

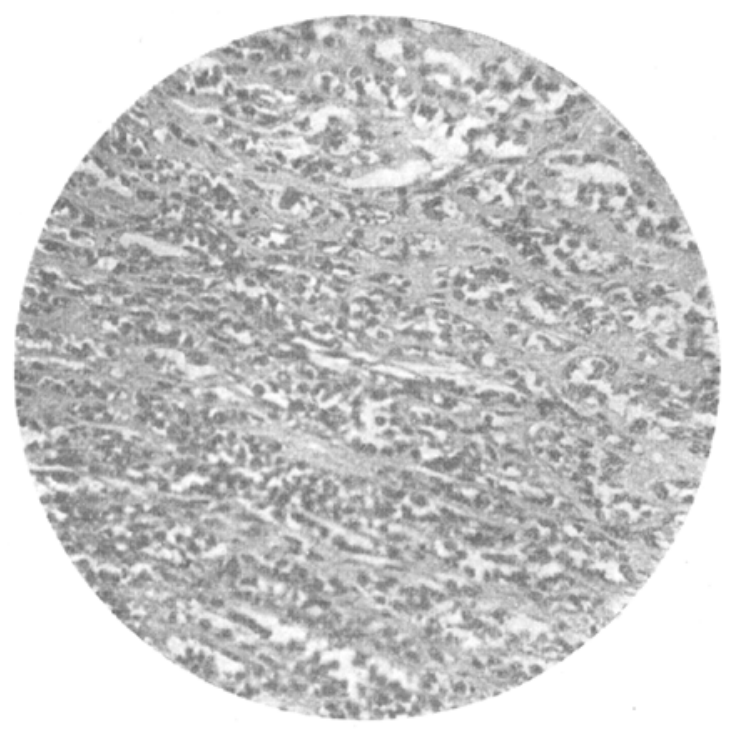

Figur 5.

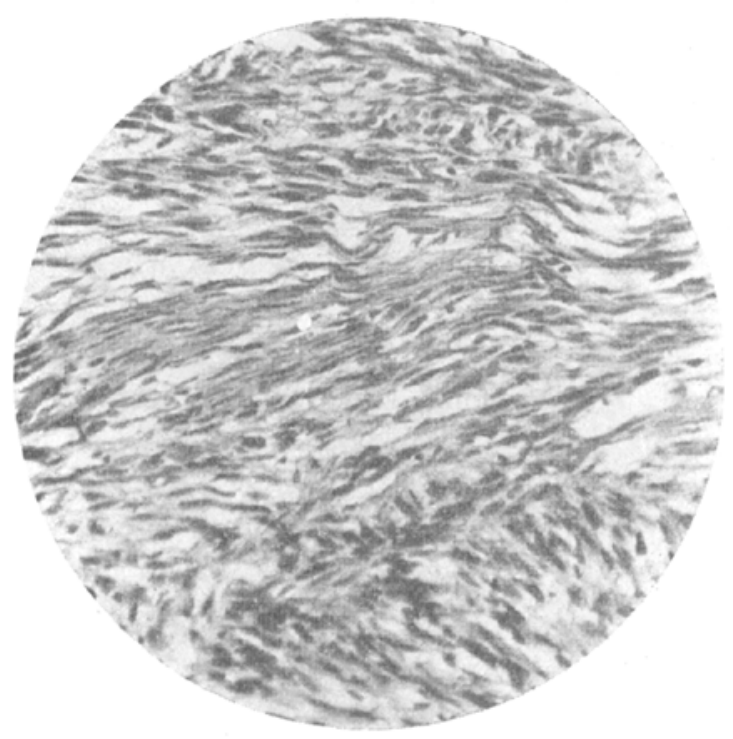

zellige Infiltration. Die Uteruswand ist durch den Tumor verdünnt und besteht aus einem dichten fibromuskulären Bindegewebe. Ein ähnliches Gewebe bildet die Kapsel des Tumors. 
Figar 6.

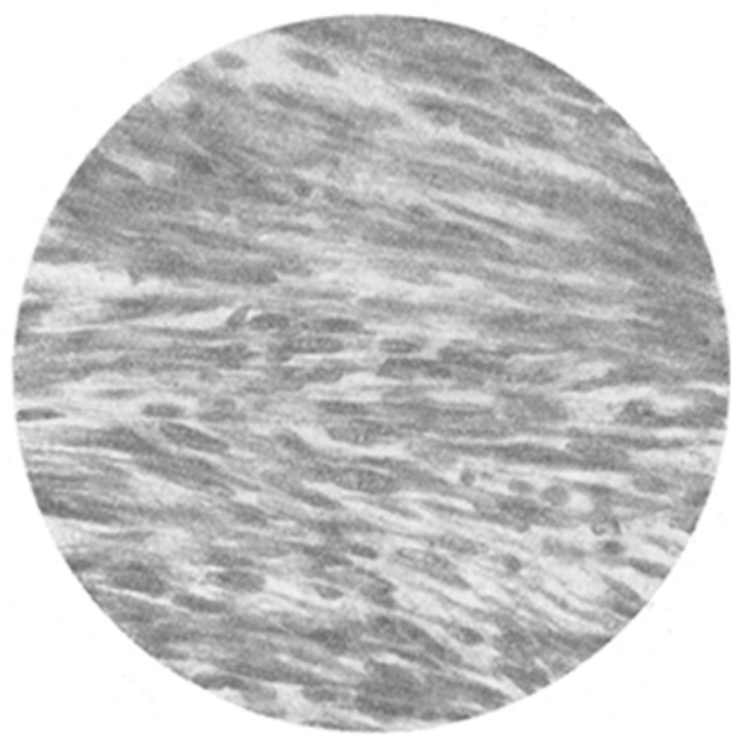

Figur 7.

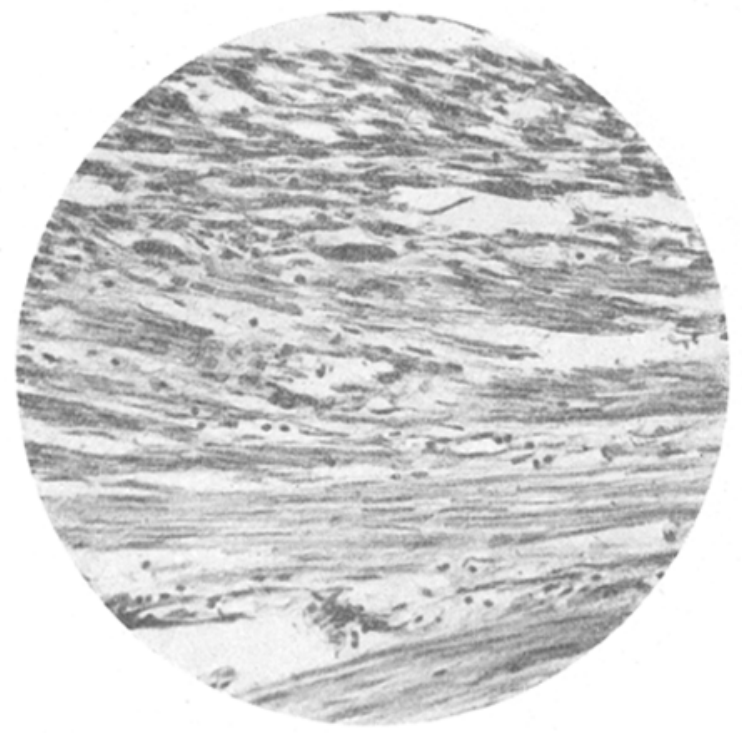

a) Harter Theil des Tumors.

Dieser Theil zeigt das Aussehen eines Fibromyoms; bei welchem das fibröse Gewebe eine hyaline Veränderung zeigt und die Muskelfasern fast ganz verschwunden sind. In vielen Abschnitten 
kann man keine Structur mehr erkennen, man sieht ein homogenes und fast kernloses Gewebe.

b) Theil des Tumors, welcher makroskopisch einem Fibromyom gleicht.

Hier sieht man ein Stroma von fibrösem Gewebe, in dessen Maschen man kleine Rundzellen, im Durchschnitt zum Theil schräg getroffen, bemerkt. Die Zellen scheinen von dem fibrösen Gewebe auszugehen (Fig. 4). Dieselben sind in Grösse und Form ziemlich gleich und das Stroma ist regelmässig; dazwisehen aber sieht man breitere Bänder von fibrösem, anscheinend degenerirtem Gewebe. Daselbst sind auch zahlreiche dünnwandige Gefässe. Die ganz gleiche Structur findet man auch in einigen Theilen des secundären Tumors (Fig. 8).

c) Der homogene Theil des Tumors.

Dieser scheint ganz cellulär zu sein; van Gieson's Färbung zeigt eine gleichmässige gelbe Beschaffenheit und keine Spur von fibrösem Stroma. Die Zellen aber sind wie bei einem Uterusmyom angeordnet. Das Präparat gleicht einem Spindelzellen-Sarkom, bei welchen die Zellen nach Art der Uterus-Muskeln gelagert sind. Die Zellen erscheinen kürzer als die der normalen Muskulatur, die Kerne breiter und oft granulirt (Fig. 6). Einige dieser Kerne sind sehr gross, unregelmässig und dunkel gefärbt. Zahlreiche Lymphspalten aber sehr wenig Blutgefässe durchziehen das Zellengewebe. Im Centrum des Tumors, da wo die Erweichung am stärksten ist, besteht derselbe aus nekrotisirtem Gewebe und zerfallenen Leukocyten. Viele dieser nekrotisirten Zellen können bis weit in den harten Theil des Tumors verfolgt werden. Andererseits können Theile des harten Tumors stellenweise in dem Tumor c gefunden werden. Solche Spuren können mit van Gieson's-Färbung hie und da in dem Präparat erkannt wurden.

Es scheint sich hier um Uebergänge zu handeln zwischen dem rein cellulären Theil und dem Bindegewebe-Theil des Tumors. Die cellulären Elemente haben offenbar die Neigung zu proliferiren und zwar auf Kosten des Bindegewebes. Da, wo der Tumor die Uteruswand durchsetzt, ist der maligne Charakter deutlich entschieden. Die unregelmässigen Zellen dringen in das normale Gewebe ein und verursachen eine leichte kleinzellige Infiltration der Muskulatur, so zu sehen auf Fig. 7 .

Der secundäre am Arm aufgetretene Tumor zeigt eine bemerkenswerthe Aehnlichkeit mit dem primären Tumor. Der fibröse 
Theil dieses Tumors ist sehr dem unter b) beschriebenen Theilen des primären Tumors ähnlich. Fig. 8. Aber die Gefässentwickelung ist viel reichlicher als im primären Tumor. Hie und da sieht man Stränge von hellgefärbtem, homogenem Gewebe. Die Hauptmasse des secundären Tumors zeigt die gleiche Muskelanordnung wie der primäre Tumor. Die Zellen gleichen denjenigen der normalen Uterusmuskulatur, aber die Kerne erscheinen grösser und unregelmässig gestaltet. Hie und da sieht man sehr grosse dunkelgranulirte Kerne. Im keinem Theil des secundären Tumors fehlt das fibröse. Stroma ganz, wie solches in dem homogenen Theil des primären Tumors bemerkt wurde.

\section{Figur 8.}

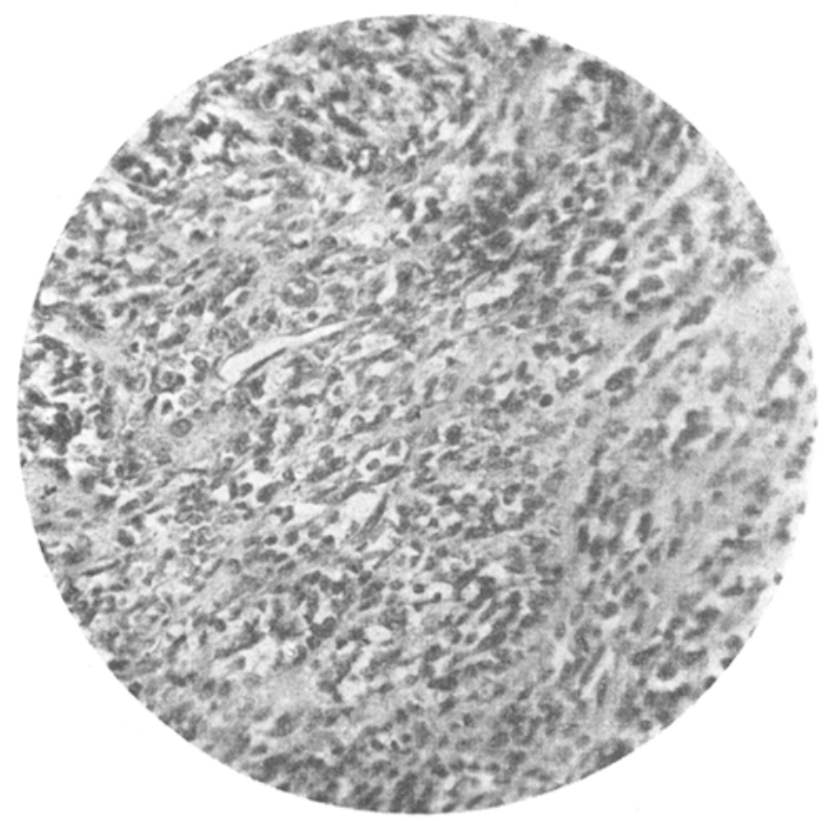

Das Studium der Lebensgeschichte der Fibromyome, besonders ihrer malignen Veränderungen ist wichtig in Bezug auf Operationen. Die alte Lehre, dass diese Tumoren harmlos sind, dass sie durch die Menopause günstig beeinflusst und selten lebensgefährlich werden, dürfte zukünftig zu Gunsten einer mehr aktiven Behandlung modificirt werden.

Trotzdem geht Noble zu weit, wenn er behauptet, man soll Fibromyome so frisch als möglich exstirpiren. Mit anderen Worten, dass das Princip der frühzeitigen Operation, welches jetzt allgemein 
266 Kynoch, Zwei Fälle von malignem Fibromyom des Uterus.

für Ovarialtumoren gilt, ebenso für die Behandlung der Fibroidtumoren gelten soll.

Am besten wird das Interesse der Myom-Patientinnen dadurch gewahrt, dass man sorgfältig das Wachsthum und die Grösse der Tumoren zur Zeit der Menopause beobachtet. Im Falle eines Wachsthums würde die Operation unumgänglich nothwendig sein, da die degenerativen Veränderungen der Fibromyome relativ häufig

Figur 9.

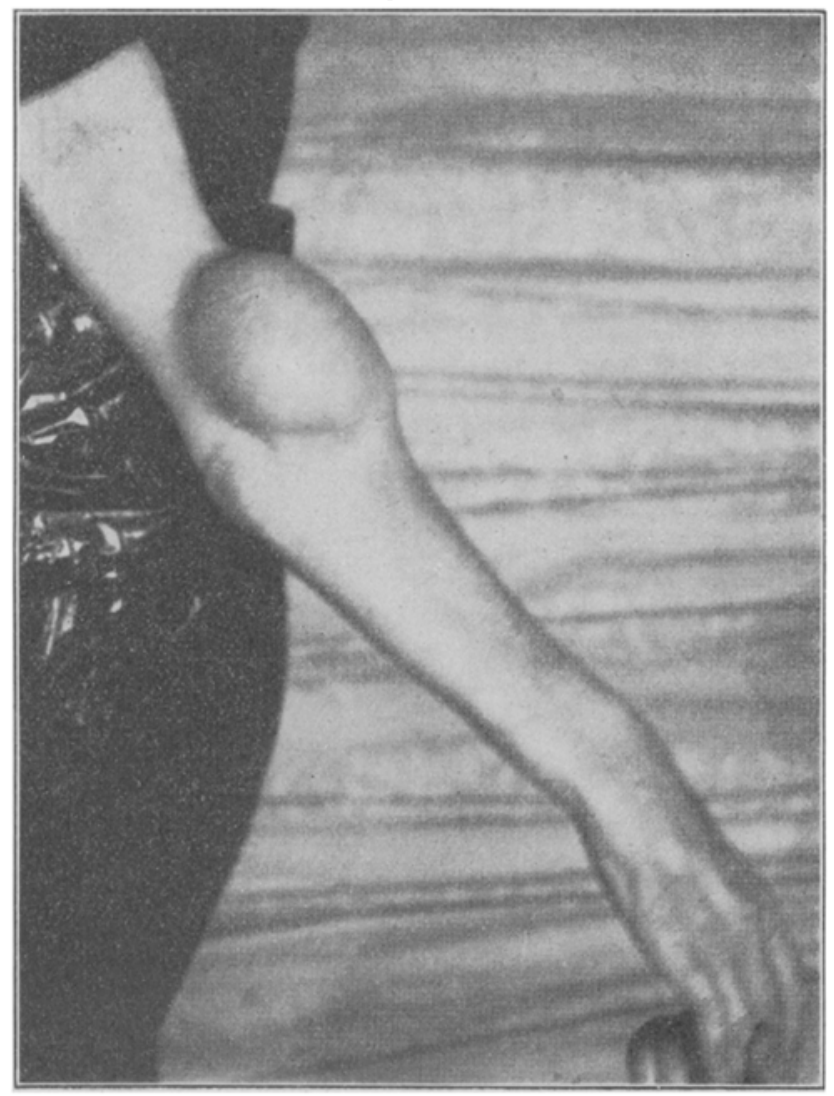

sind. Die Frage des relativen Werthes von Panhysterectomie oder supra-vaginaler Amputation ist noch ungelöst. Von Winckel begünstigt die letztere, weil eine nachträgliche Carcinom-Entwickelung in dem Cervical-Stumpf und eine maligne Veränderung der Fibromyome im Grossen und Ganzen selten sind. Anderseits sagt Richelt, dass alles das auch zu Gunsten der Panhysterectomie angeführt werden kann, und dass schon die Möglichkeit eines nachfolgonden Cervieal-Krebses zur. Wahl der Panhysterectomie zwinge. 